\title{
A comparison of visual and quantitative methods to identify interstitial lung abnormalities
}

\author{
Corrine R. Kliment ${ }^{1}$, Tetsuro Araki ${ }^{2}$, Tracy J. Doyle ${ }^{1}$, Wei Gao ${ }^{3}$, Josée Dupuis ${ }^{3,4}$, Jeanne C. Latourelle ${ }^{5,6}$, \\ Oscar E. Zazueta1', Isis E. Fernandez ${ }^{1}$, Mizuki Nishino ${ }^{2,7}$, Yuka Okajima², James C. Ross ${ }^{8,9}$, Raúl San José Estépar ${ }^{7,9}$, \\ Alejandro A. Diaz ${ }^{1,10}$, David J. Lederer ${ }^{11,12}$, David A. Schwartz ${ }^{13}$, Edwin K. Silverman ${ }^{1,8}$, Ivan O. Rosas ${ }^{1}$, \\ George R. Washko ${ }^{1,7}$, George T. O'Connor ${ }^{4,14}$, Hiroto Hatabu ${ }^{2,7}$ and Gary M. Hunninghake ${ }^{1,7^{*}}$
}

\begin{abstract}
Background: Evidence suggests that individuals with interstitial lung abnormalities (ILA) on a chest computed tomogram (CT) may have an increased risk to develop a clinically significant interstitial lung disease (ILD). Although methods used to identify individuals with ILA on chest CT have included both automated quantitative and qualitative visual inspection methods, there has been not direct comparison between these two methods. To investigate this relationship, we created lung density metrics and compared these to visual assessments of ILA.
\end{abstract}

Methods: To provide a comparison between ILA detection methods based on visual assessment we generated measures of high attenuation areas (HAAs, defined by attenuation values between -600 and -250 Hounsfield Units) in $>4500$ participants from both the COPDGene and Framingham Heart studies (FHS). Linear and logistic regressions were used for analyses.

Results: Increased measures of HAAs (in $\geq 10 \%$ of the lung) were significantly associated with ILA defined by visual inspection in both cohorts $(P<0.0001)$; however, the positive predictive values were not very high $(19 \%$ in COPDGene and $13 \%$ in the FHS). In COPDGene, the association between HAAs and ILA defined by visual assessment were modified by the percentage of emphysema and body mass index. Although increased HAAs were associated with reductions in total lung capacity in both cohorts, there was no evidence for an association between measurement of HAAs and MUC5B promoter genotype in the FHS.

Conclusion: Our findings demonstrate that increased measures of lung density may be helpful in determining the severity of lung volume reduction, but alone, are not strongly predictive of ILA defined by visual assessment. Moreover, HAAs were not associated with MUC5B promoter genotype.

Keywords: High attenuation areas, Idiopathic pulmonary fibrosis, Interstitial lung disease, Interstitial lung abnormalities (ILA), MUC5B

\section{Background}

Although idiopathic pulmonary fibrosis (IPF), the most common and severe form of interstitial lung disease (ILD), has historically been unresponsive to pharmacotherapy $[1,2]$, recent studies have finally demonstrated that two medical therapies $[3,4]$ can reduce the rate of decline in lung function, particularly when started early

\footnotetext{
* Correspondence: ghunninghake@partners.org

${ }^{1}$ From the Pulmonary and Critical Care Division, Brigham and Women's

Hospital, Harvard Medical School, Boston, MA, USA

${ }^{7}$ Center for Pulmonary Functional Imaging, Brigham and Women's Hospital, Boston, MA, USA

Full list of author information is available at the end of the article
}

in the course of disease [5]. These findings provide an important motivation to identify patients in early stages of disease.

Accumulating evidence suggest that interstitial lung abnormalities (ILA) identified on chest imaging may identify groups of subjects at risk to develop clinically significant ILD in general [6], and IPF in particular $[7,8]$. Support for this statement comes evidence that participants with ILA are more likely to have respiratory symptoms $[9,10]$ and physiologic decrements (e.g. reduced lung volumes [9-11], exercise capacity [12], and diffusion capacity of carbon monoxide 
[DLCO]) $[7,10]$ suggestive of, but less severe than, those apparent in patients with IPF [13]. In addition, multiple studies have identified radiologic progression in participants with ILA [7, 8, 14], a usual interstitial pneumonia (UIP) pattern on chest computed tomograms (CTs) in some participants with ILA [7-9, 11, 14], and progression from a non-UIP pattern to a UIP pattern in small numbers of subjects $[7,8]$. Finally, the genetic factor most strongly associated with IPF (MUC5B promoter genotype) [15] has been similarly associated with ILA in the Framingham Heart Study (FHS) [10].

While these findings suggest the potential importance of identifying ILA, and comparisons between quantitative and visual assessments of clinically established pulmonary fibrosis have been performed [16-18], there has been no published data on the relationship between quantitative and visual assessments for ILA identification. In particular, while all studies to date have utilized chest $\mathrm{CTs}$ and most have relied on qualitative visual assessment [7-10, 14], one study utilized a quantitative assessment of lung density to identify ILA [11]. Assessing lung density as a method of identifying ILA is attractive as it could be automated, it could provide a continuous metric of the degree of abnormality, and it would be compatible with methods commonly used to identify and quantify the extent of emphysema in research studies [19]. To compare these two methods, we created lung density metrics and compared these to visual assessments of ILA in two large cohorts $[9,10]$.

\section{Methods}

\section{Study design}

Protocols for enrollment and phenotyping for both the COPDGene and the Framingham Heart Study (FHS) participants have been described previously $[9,10]$. In brief, the COPDGene cohort analyzed in this article includes 2508 non-Hispanic White ( $n=1867,74 \%)$, and AfricanAmerican $(n=641,26 \%)$ smokers (with at least 10 pack years of smoking) between the ages of 45 and 80, who were enrolled into COPDGene at 21 clinical centers from November of 2007 to April of 2010. The FHS cohort included in this article includes 2764 mostly non-Hispanic white adult men and women from the Third Generation and Offspring Cohorts who participated in FHS MultiDetector Computed Tomography 2 (FHS-MDCT2) Study. The COPDGene study (including this ancillary study) was approved by the institutional review boards (IRBs) of all participating centers and FHS-MDCT2 study (and this ancillary study) was approved by the Boston University and the Brigham and Women's Hospitals' IRBs (2010P000996 and 2007P000554 for the FHS and COPDGene, respectively). All included participants in both cohorts provided written informed consent, including consent for the use of their DNA in genetic studies.

\section{Chest $\mathrm{CT}$ acquisition protocols}

In COPDGene, the CT acquisition protocol used was as follows: $120 \mathrm{kVp}, 200 \mathrm{mAs}$, and $0.5 \mathrm{~s}$ rotation time for General Electric (GE) LightSpeed-16, GE VCT-64, Siemens Sensation-16, Siemens Sensation-64, Philips 40slice, and Philips 64-slice scanners. Images were reconstructed using a standard algorithm at $0.625 \mathrm{~mm}$ slice thickness and $0.625 \mathrm{~mm}$ intervals for GE scanners. Siemens CT images were reconstructed using a B31f algorithm at 0.625 (Sensation-16) or $0.75 \mathrm{~mm}$ slice thickness and $0.5 \mathrm{~mm}$ intervals. Reconstruction of Philips images was performed by using $B$ algorithm at $0.9 \mathrm{~mm}$ slice thickness and $0.45 \mathrm{~mm}$ intervals. In the FHS, CT images were acquired with a General Electric Discovery VCT 64-slice PET/CT scanner (GE Healthcare) using a MA determined by subject weight $(300 \mathrm{~mA}$ for subjects less than $220 \mathrm{lbs}, 350 \mathrm{mG}$ for subjects equal to or greater than $220 \mathrm{lbs}) 120 \mathrm{Kv}$, and a gantry rotation time of $0.35 \mathrm{~s}$. Raw data was collected using a $210^{\circ} \mathrm{scan}$ reconstruction algorithm and a detector width of $0.625 \mathrm{~mm}$. Images were reconstructed with a $50 \mathrm{~cm}$ field of view (FOV).

\section{Thoracic chest $\mathrm{CT}$ analysis \\ Visual assessment}

The methods for the visual assessment of chest CTs in both COPDGene and FHS have been described previously $[9,10]$. Chest CTs were evaluated by three readers using a sequential reading method as previously described [20]. ILA were defined as nondependent changes (to exclude the effect of atelectasis as prone images were not available) affecting $>5 \%$ of any lung zone including, nondependent ground-glass or reticular abnormalities, diffuse centrilobular nodularity, nonemphysematous cysts, honeycombing, or traction bronchiectasis. CTs with either focal or unilateral ground-glass attenuation, focal or unilateral reticulation, or patchy ground-glass abnormality ( $<5 \%$ of the lung) were considered indeterminate.

\section{Quantitative assessment}

Image analysis was performed on inspiratory CT scans using Pulmonary Workstatins 2 and PLUS (VIDA Diagnostics, Iowa City, IA) at the Core Imaging Center of the COPDGene study to quantify the percentage of the lung occupied by high attenuation areas (HAAs, defined by attenuation values between -600 and -250 Hounsfield Units [HUs]) [11]. While this method includes lung segmentation of both the parenchyma and central vasculature, these vessels are ultimately excluded in quantitation based on the 
attenuation values of blood ( $40 \mathrm{HUs}$ depending on the hematocrit). Airway Inspector (http://airwayinspector.acil-bwh.org) was used to quantify the total lung capacity and percentage of emphysematous lung (at [-]950, in COPDGene) [19].

\section{Statistical analysis}

In both COPDGene and the FHS, the percentage of HAAs was evaluated as both a continuous and as a binary variable selected on the basis of a prior presentation [11] (e.g. $\geq 10 \%$ or $<10 \%$ and $\geq 6.4 \%$ or $<6.4 \%[\geq 6.4 \%$ corresponds to the $\geq$ upper $95^{\text {th }}$ percentile] of the lung occupied by HAAs in COPDGene). To provide a complete comparison between methods, when ILA was the primary outcome we assessed controls as those that both included and excluded participants indeterminate for ILA by visual assessment. In COPDGene, covariate adjustment and statistical analyses were performed as described using Statistical Analysis Software version 9.2 (SAS Institute, Cary, NC). In the FHS, all analyses accounted for familial relationship using generalized linear mixed effect models as previously described [21] and were performed using $\mathrm{R}$ version 2.9 [22]. All genetic analyses were performed using additive genetic models
[10]. Reported $P$ values are two-sided and those $<0.05$ were considered statistically significant.

\section{Results}

\section{Population characteristics of ILA by characterization} method

Population characteristics of participants with ILA, defined by both visual assessment and a threshold of HAAs for both the COPDGene and FHS, are presented in Table 1. Population characteristics of participants without ILA and indeterminate for ILA defined by visual assessment and the interobserver variability of ILA assessments in both the COPDGene and FHS have been presented previously $[9,10]$. The most strikingly consistent differences between the classification methods of ILA are that participants with ILA defined by HAAs tended to have an increased BMI and greater reductions in total lung capacity (TLC) in both the COPDGene and FHS cohorts when compared to participants with ILA classified by visual assessment.

\section{COPDGene}

In COPDGene, of the 2416 chest CTs previously assessed for the presence of ILA [9], quantitative assessment of

Table 1 Baseline characteristics of COPDGene and Framingham Heart Study (FHS) participants with Interstitial Lung Abnormality (ILA) by identification method

\begin{tabular}{|c|c|c|c|c|}
\hline \multirow{4}{*}{$\begin{array}{l}\text { Variable }^{a} \\
\text { Demographic parameters }\end{array}$} & \multicolumn{4}{|c|}{ Number (\%) or Median (standard deviation) where appropriate } \\
\hline & \multicolumn{2}{|l|}{ COPDGene } & \multicolumn{2}{|l|}{ FHS } \\
\hline & ILA by Visual Assessment & ILA by $\mathrm{HAAs}^{\mathrm{b}}$ & ILA by Visual Assessment & ILA by $H A A s^{b}$ \\
\hline & $n=194(8 \%)$ & $n=26(1 \%)$ & $n=177(7 \%)$ & $n=46(2 \%)$ \\
\hline Age (years) & $64(10)$ & $62(8)$ & $70(12)$ & $65(13)$ \\
\hline Gender (female) & $101(52 \%)$ & $14(54 \%)$ & $89(50 \%)$ & $19(41 \%)$ \\
\hline Race (African-American) & $56(29 \%)$ & $14(54 \%)$ & - & - \\
\hline Body Mass Index & $28(7)$ & $33(4)$ & $28(5)$ & $32(5)$ \\
\hline Pack years of smoking & $44(27)$ & $42(26)$ & $26(20)$ & $18(15)$ \\
\hline Current Smoker & $97(50 \%)$ & $18(69 \%)$ & $17(10 \%)$ & $5(11 \%)$ \\
\hline \multicolumn{5}{|l|}{ Spirometric Parameters } \\
\hline $\mathrm{FEV}_{1}(\% \text { of predicted })^{\mathrm{d}}$ & $81 \%(21)$ & $80 \%(32)$ & $98 \%(17)$ & $96 \%(16)$ \\
\hline FVC (\% of predicted $)^{d}$ & $88 \%(17)$ & $78 \%(23)$ & $101 \%(15)$ & $96 \%(15)$ \\
\hline FEV1/FVC \% ${ }^{d}$ & $71 \%(14)$ & $81 \%(18)$ & $97 \%(9)$ & $100 \%(7)$ \\
\hline DLCO (\% of predicted $)^{e}$ & - & - & $86 \%(14)$ & $86 \%(17)$ \\
\hline \multicolumn{5}{|l|}{ Chest $C T$ parameters } \\
\hline Total Lung Capacity (TLC) ${ }^{f}$ & $5.0(1.4)$ & $3.5(1.4)$ & $4.6(1.2)$ & $3.8(0.9)$ \\
\hline TLC \% of predicted ${ }^{f}$ & $95 \%(20)$ & $57 \%(17)$ & $79 \%(17)$ & $64 \%(14)$ \\
\hline \multicolumn{5}{|c|}{ 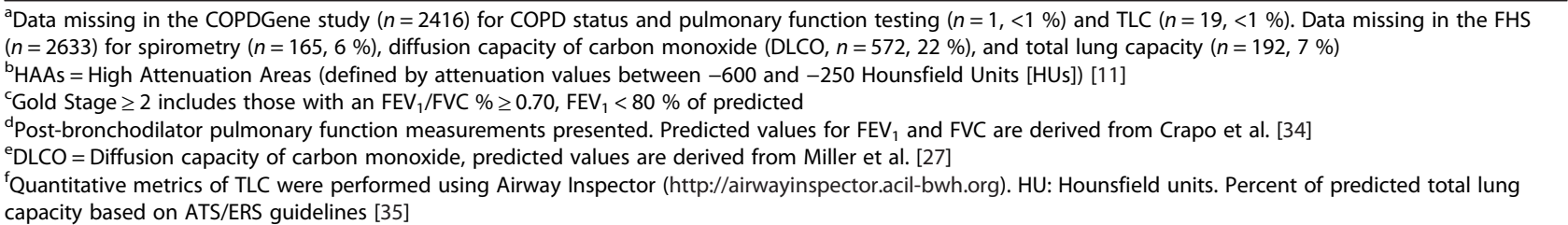 } \\
\hline
\end{tabular}


HAA could be measured in 2093 (87 \%) participants. HAAs were significantly associated with ILA in analyses that excluded participants indeterminate for ILA status (for each $1 \%$ increase in HAAs a participant had a 2.3fold increase in their odds to have ILA, $95 \%$ confidence interval $[\mathrm{CI}] 2.0-2.6, P<0.0001)$ and in analyses including participants indeterminate for ILA as controls (for each $1 \%$ increase in HAAs a participant had a 1.3-fold increase in their odds to have ILA, $95 \%$ confidence interval [CI] $1.2-1.4, P<0.0001)$. In addition, increases in HAAs were associated with reductions in total lung capacity (TLC) both in analyses of all participants (after adjusting for covariates including age, sex, race, body mass index [BMI], smoking behavior, the percent of emphysema and COPD status) and in analyses limited to those with ILA (after adjusting for covariates). For all participants, for each $1 \%$ increase in HAAs a participant was predicted to have a $351 \mathrm{ml}$ decrease in TLC (95 \% CI 324-378 ml, $P<0.0001$ ). For those with ILA, for each $1 \%$ increase in HAAs a participant was predicted to have a $278 \mathrm{ml}$ decrease in TLC (95\% CI 216-340 ml, $P<0.0001)$. However, as noted in Fig. 1, the difference between the median value of the percentage of

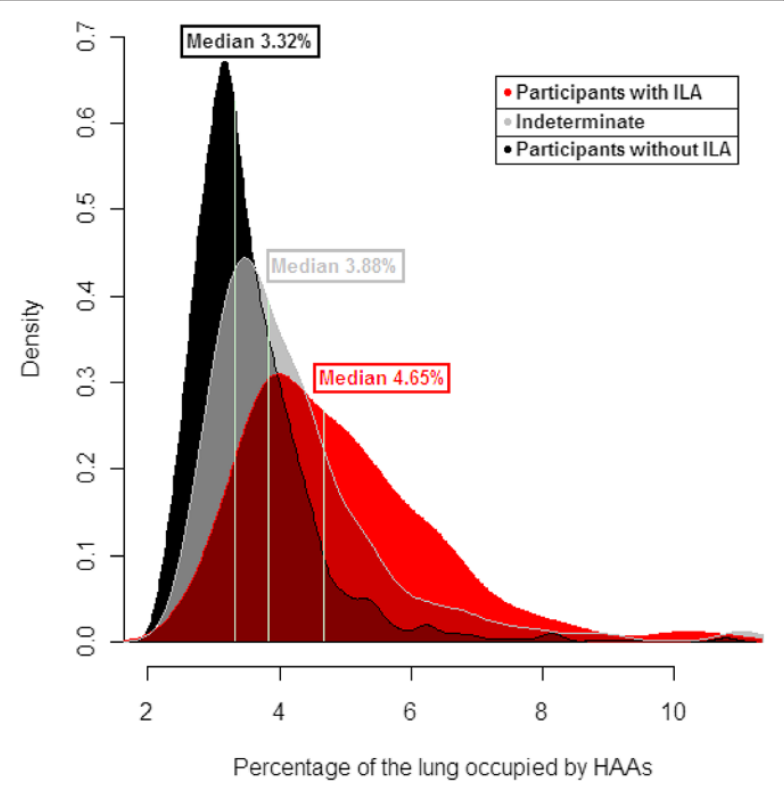

Fig. 1 A density plot of the percentage of the lung occupied by High Attenuation Areas (HAAs, chest CT attenuation values between -600 and $-250 \mathrm{HU}$ ) in participants with ILA (in red, $n=163$ ), in participants indeterminate for ILA (in gray, $n=757$ ), and in the participants without ILA (in black, $n=1173$ ). Despite the differences in numbers between the groups for each category (defined by color) the area under the curve is normalized to a density of 1 which gives a sense of the relative spread of the data between categories. The percentage of lung occupied by various HAA thresholds is listed on the x-axis. The density of subjects at various HAA thresholds is listed on the $y$-axis the lung occupied by HAAs among participants with ILA, compared to those without ILA, was $<1.4 \%$. Among participants with ILA, the median value of HAAs was $4.6 \%$ ranging between 2.7 and $16.5 \%$. Among participants without ILA, the median value of HAAs was $3.3 \%$ ranging between 2.2 and $10.8 \%$.

Next, we assessed the ability of HAA thresholds to predict the presence of ILA by visual assessment in COPDGene. As noted in Table 2, although there was significant evidence for a correlation between ILA defined by visual assessment and those having $\geq 10 \%$ of the lung occupied by HAAs $(P=0.03)$, the agreement between methods was slight (Kappa $=0.03)$. The positive predictive value of having $\geq 10 \%$ HAAs in the prediction of ILA by visual assessment in COPDGene was $19 \%$. We considered an alternate threshold of lung density $\left(\geq 6.4 \%\right.$, corresponding to the upper $95^{\text {th }}$ percent of HAAs in COPDGene), and noted increased evidence for a correlation between ILA defined by visual assessment and lung density methods $(P<0.0001)$. However, the agreement between methods remained modest (Kappa $=$ 0.13). The best performing threshold of HAAs in the prediction of ILA defined by visual assessment (c-statistic 0.76 ) was obtained with an HAA threshold of $\geq 3.6 \%$ (which corresponds to the $50^{\text {th }}$ percentile of HAAs in COPDGene).

There is evidence that the association between HAAs and ILA defined by visual assessment was modified by the percentage of emphysema $(P=<0.0001)$ and the BMI $(P=0.03)$ of participants. For example, among participants with $<5 \%$ emphysema, for each $1 \%$ increase in HAAs a participant had a 1.2-fold increase in the odds of having ILA (95 \% CI 1.1-1.3, $P<0.0001$ ). Whereas, among participants with $\geq 5 \%$ emphysema, for each $1 \%$ increase in HAAs a participant had a 3.8-fold increase in the odds of having ILA (95\% CI 2.3-6.0, $P<0.0001$ ). Among participants with a BMI $\geq 30$, for each $1 \%$ increase in HAAs a participant had a 1.9-fold increase in the odds of having ILA (95\% CI 1.6-2.3, $P<0.0001$ ). Whereas, among participants with a BMI $<30$, for each $1 \%$ increase in HAAs a participant had a 3.1-fold increase in the odds of having ILA (95\% CI 2.5-4.0, $P<0.0001$ ). Figure 2 demonstrates an example of a participant with ILA defined by visual assessment on the background of emphysema but with $<10 \%$ HAAs, and an example of a participant without ILA defined by visual assessment and an increased BMI with $\geq 10 \%$ HAAs. As apparent in Fig. 2 (and noted in the data above), an increase in the percentage of emphysema appears to reduce the sensitivity of HAA thresholds for the detection of ILA by visual assessment, while an increased BMI alone can result in an increase in the percentage of HAAs even when there is minimal visual evidence for ILA on chest CT imaging. 
Table 2 Comparison of methods for the detection of interstitial lung abnormalities in COPDGene and Framingham Heart Study (FHS) participants

\begin{tabular}{|c|c|c|c|c|}
\hline COPDGene & $\mathrm{HAAs}<10 \%^{\mathrm{a}}$ & $\mathrm{HAAs} \geq 10 \%^{\mathrm{a}}$ & HAAs $<95^{\text {th }}$ percentile $(\mathrm{HAA}<6.44 \%)^{\mathrm{a}}$ & HAAs $\geq 95$ percentile $(\mathrm{HAA} \geq 6.44 \%)^{\mathrm{a}}$ \\
\hline$\overline{\text { No ILA }}{ }^{a}$ & 1909 & 21 & 1850 & 80 \\
\hline \multirow[t]{2}{*}{ ILA } & 158 & 5 & 139 & 24 \\
\hline & \multicolumn{2}{|c|}{ Kappa $0.03, p=0.03$} & \multicolumn{2}{|l|}{ Kappa $0.13, p<0.0001$} \\
\hline Framingham Heart Study & HAAs $<10 \%$ & HAAs $\geq 10 \%$ & HAAs $<95^{\text {th }}$ percentile & HAAs $\geq 95$ percentile \\
\hline No ILA ${ }^{a}$ & 1987 & 306 & 2188 & 105 \\
\hline \multirow[t]{2}{*}{ ILA } & 104 & 46 & 132 & 18 \\
\hline & \multicolumn{2}{|c|}{ Kappa $0.11, p=<0.0001$} & \multicolumn{2}{|l|}{ Kappa $0.08, p<0.0001$} \\
\hline \multicolumn{5}{|l|}{ COPDgene Cohort: } \\
\hline HAAs at $10 \%$ & Sensitivity $3 \%$ & Specificity $99 \%$ & PPV $19 \%$ & NPV $92 \%$ \\
\hline HAAs at $95^{\text {th }} \%$ & Sensitivity $15 \%$ & Specificity $96 \%$ & PPV $23 \%$ & NPV $93 \%$ \\
\hline \multicolumn{5}{|l|}{ Framingham Cohort: } \\
\hline HAAs at $10 \%$ & Sensitivity $31 \%$ & Specificity $87 \%$ & PPV $13 \%$ & NPV $95 \%$ \\
\hline HAAs at $95^{\text {th }} \%$ & Sensitivity $12 \%$ & Specificity $95 \%$ & PPV $15 \%$ & NPV $94 \%$ \\
\hline
\end{tabular}

${ }^{a}$ HAAs high attenuation areas (defined by the percentage of the lung occupied by high attenuation areas between -600 and -250 Hounsfield Units) [11]

${ }^{b}$ Number of subjects grouped as "no ILA" that are classified as indeterminate: COPDgene: HAA $<10 \%-n=739, \mathrm{HAAs} \geq 10 \%-n=18, \mathrm{HAAs}<95 \%-n=698$, HAAs $\geq 95 \%-n=59$

"Number of subjects grouped as "no ILA" that are classified as indeterminate: Framingham: HAA $<10 \%-n=805, \mathrm{HAAs} \geq 10 \%-n=184, \mathrm{HAAs}<95 \%-n=926$, HAAs $\geq 95 \%-n=63$

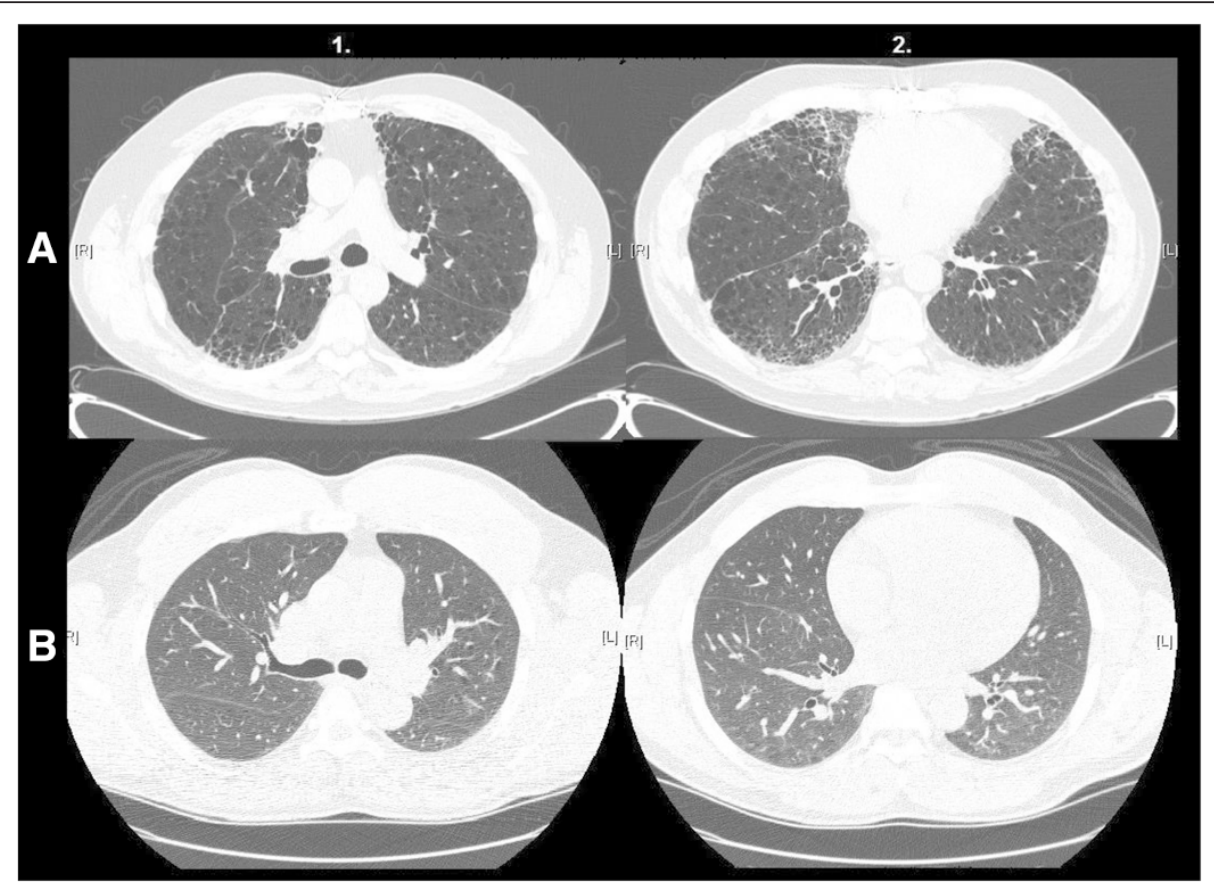

Fig. 2 On the vertical axis we present representative examples of (a) a subject with interstitial lung abnormalities identified by visual assessment but has less than $10 \%$ of the lung with chest CT attenuation values between -600 and $-250 \mathrm{HU}(\mathrm{HAA})(7.5 \% \mathrm{HAA})$ and (b) a subject having $>10 \%$ of the lung with chest $C T$ attenuation values between -600 and $-250 \mathrm{HU}$ (HAA) (10.8 \%) but not identified as having interstitial lung abnormalities identified by visual assessment. Each row represents data from a single subject. On the horizontal axis we present axial high resolution chest computed tomographic (HRCT) images (1 [approximately at the level of the carina] and 2 [approximately at the level of the right inferior pulmonary vein]) 


\section{Framingham Heart Study (FHS)}

As a prior study utilizing HAAs in the identification of ILA was performed in a general population sample [11], we additionally analyzed the FHS and compared the association between thresholds of HAAs and ILA defined by visual assessment in 2443 (93\%) of the previously assessed 2633 chest CTs, where this measurement could be obtained.

As noted in Table 2, and comparable to our findings in COPDGene, although there was significant evidence for a correlation between ILA defined by visual assessment and those having $\geq 10 \%$ of the lung occupied by HAAs $(P=<0.0001)$, the agreement between methods was slight $(\mathrm{Kappa}=0.11)$. Comparable to results from COPDGene, increases in HAAs were associated with reductions in TLC both in analyses of all participants (after adjusting for covariates including age, sex, race, BMI, and smoking behavior, for each $1 \%$ increase in HAAs a participant was predicted to have a $233 \mathrm{ml}$ decrease in TLC, $95 \%$ CI $223-243 \mathrm{ml}, P<0.0001)$ and in analyses limited to those with ILA (after adjusting for covariates, for each $1 \%$ increase in HAAs a participant was predicted to have a $214 \mathrm{ml}$ decrease in TLC, $95 \%$ CI 179-249 $\mathrm{ml}, P<0.0001)$. However, the positive predictive value of having $\geq 10 \%$ HAAs in the prediction of ILA by visual assessment in the FHS was $13 \%$. Similar evidence was noted when we considered an alternate threshold of lung density $(\geq 6.4 \%$, Kappa $0.08, P<0.0001)$. The positive predictive value of having $\geq 6.4 \%$ HAAs in the prediction of ILA by visual assessment in the FHS was $15 \%$.

Finally, based on a prior association between $M U C 5 B$ promoter genotype (rs3570950) and ILA defined by visual assessment in the FHS [10], we compared the association between $M U C 5 B$ promoter genotype and ILA defined by various thresholds of HAAs and continuous measures of HAAs. After adjusting for covariates including age, sex, body mass index, and smoking behavior, there was no evidence for an association between $M U C 5 B$ promoter genotype and having $\geq 10 \%$ of the lung occupied by HAAs (OR 1.1, $95 \%$ CI $0.9-1.5, P=$ $0.33)$, or when we considered an alternate threshold of lung density ( $\geq 6.44 \%$ in the FHS, OR $1.1,95 \%$ CI $0.7-$ 1.6, $P=0.75$ ). Finally, after adjusting for covariates, there was no evidence for an association between $M U C 5 B$ promoter genotype and continuous measures of HAAs in the FHS $(P=0.31)$.

\section{Discussion}

Our findings demonstrate that, although individuals with an increased percentage of the lung occupied by regions of high density are more likely to have interstitial lung abnormalities defined by visual assessment in both the COPDGene and the FHS cohorts, lung density thresholds do not appear to be strongly predictive of ILA defined by visual assessment. Although ILA defined by visual assessment and ILA defined by lung density thresholds both predict a phenotype that is associated with reduced total lung capacity, ILA defined by lung density thresholds are not associated with $M U C 5 B$ promoter genotype.

As information accrues that suggests that the presence ILA on chest CT, in some cases, may define groups that are at an increased risk for the development of clinically evident pulmonary fibrosis [6], consistency in ILA detection across studies will be important to allow for accurate comparisons. Automated methods of lung abnormality detection are attractive in that they could provide a rapid, quantifiable, and reproducible method (at least across similar scanners and scanning protocols) [23] to determine if a person is at an increased risk for pulmonary fibrosis. Unfortunately, our findings suggest that most cases of ILA defined by visual assessment would be missed by detection methods based on classification using lung density alone. Given that the primary purpose of this research is to identify groups at an increased risk for pulmonary fibrosis, it is important to note that although both methods identify groups with reduced lung volumes (an effect noticeably greater with ILA detection methods based on lung density), the genetic factor most strongly associated with IPF (MUC5B promoter genotype) $[15,24-28]$ is similarly associated with ILA when defined by visual assessment [10] but not with ILA when defined by thresholds of lung density. This suggests that quantitative methods for detecting ILA based on lung density may include additional phenotypes associated with reduced total lung volume measurements (based on chest CT measurements) but not suggestive of an ILD (e.g. atelectasis, reduced inspiratory volume, or increased attenuation values due to increased soft tissue as can be noted in subjects with a high BMI).

As it has been known for some time that ILD is associated with increased measures of lung density [29], it is perhaps surprising that increased measures of lung density are not strongly predictive of ILA defined by visual assessment. To explore this phenomenon further, we postulated that additional factors known to influence lung density could modify the correlation between lung density metrics and ILA defined by visual assessment. We found that both chest CT-defined metrics of emphysema (a factor defined by reduced lung density) [19] and BMI (a factor that can increase image noise and thus lung density when increased) [30] influence the correlation between measures of lung density and ILA defined by visual assessment. In short, our findings are consistent with the fact that individuals with ILA defined by visual assessment will tend to have lower measures of high attenuation areas 
when a significant amount of emphysema is present. In addition, individuals with a high BMI, even without ILA defined by visual assessment, are more likely to have increased measures of high attenuation areas. Although measures of emphysema and BMI likely do not entirely explain the lack of a strong correlation between these methods of ILA detection, our findings do suggest that factors influencing global lung density can influence the performance of lung density thresholds when classifying pulmonary parenchymal abnormalities.

Our study has a number of important limitations. First, although our study suggests that there are limitations of lung density thresholds for the classification of ILA in multiple cohorts, longitudinal follow-up of individuals with ILA defined by various methods has not been presented. Second, while our study demonstrates that automated ILA classification methods (based on global increases in regions of high attenuating areas) alone may have limitations, our study does not exclude the possibility that alternate automated ILA detection methods (including those involving lung segmentation and texture analysis) [31] could have improved classification performance. Importantly, in patients with known interstitial lung disease, radiologic discrimination and quantitation methods based on texture analysis and feature selection have demonstrated good sensitivity and specificity when compared to radiologic interpretations $[17,18,32,33]$ and have been demonstrated to be superior to lung density based detection methods [33]. Third, although evidence has been presented that suggests, in some cases, that ILA defined by visual inspection could represent an early stage of pulmonary fibrosis, it is important to note that there is currently no "gold standard" criteria for the detection of an early stage of pulmonary fibrosis. In addition, some of the radiologic features that we have used to define ILA are atypical and suggest alternate diagnoses from idiopathic pulmonary fibrosis (e.g. centrilobular groundglass nodules). Our findings do not preclude the possibility that alternate methods of visual assessment or quantitative measurement could ultimately, and more accurately, identify groups at high risk to develop pulmonary fibrosis. Fourth, it is worth noting that although specific $\mathrm{HU}$ thresholds for measurement of HAAs of the lung (between -600 and -250 HUs) exclude central pulmonary vasculature $(\sim 40 \mathrm{HU}$ depending on hematocrit), it is not known what effect residual segmental and subsegmental vasculature (which might be captured by HAA thresholds) could have on HAA quantification. Finally, although our study raises concerns about the use of high attenuation lung density thresholds alone for the classification of ILA, our study does not exclude the possibility that measures of increased lung density could provide additive clinical information. In fact, our study demonstrates that increased measures of lung density are strongly correlated with reduced lung volumes, even in analyses limited to those with ILA defined by visual assessment. Future longitudinal studies will be needed to determine if increased measures of lung density among those with ILA are associated with a greater risk for a progressive disease.

\section{Conclusion}

In conclusion, our findings demonstrate that metrics of increased lung density may be helpful in determining the severity of lung volume reduction, but alone, are insufficient in classifying interstitial lung abnormalities defined by visual assessment.

\section{Abbreviations}

HAAs: high attenuation areas; ILA: interstitial lung abnormalities; ILD: interstitial lung disease; IPF: Idiopathic pulmonary fibrosis; CT: computed tomogram; FHS: Framingham Heart studies; UIP: usual interstitial pneumonia; DLCO: diffusion capacity of carbon monoxide; HUs: Hounsfield Units; TLC: total lung capacity; BMl: Body mass index.

\section{Competing interests}

In addition to NIH grant support the following authors report these additional conflicts of interest: D.J.L. reports fees from consulting with Intermune, Gilead, Boehringer-Ingelheim, and ImmuneWorks; D.A.S. reports grants from the Veterans Administration, during the conduct of the study; other from Weitz and Luxenberg Law Firm, other from Brayton and Purcell Law Firm, other from Wallace and Graham Law Form, personal fees from Book Royalties- Medicine, Science and Dreams, outside of submitted work and additionally has a patent $6,214,806 \mathrm{~B} 1$ with royalties paid, a patent $6,740,487 \mathrm{~B} 1$ with royalties paid, a patent 10,316,191 with royalties paid, a patent 7,585,627 with royalties paid, a patent 7,785,794 with royalties paid, a patent 8,673,565 with royalties paid, a patent 61/248,505 pending, a 61/ 666,233 pending, a patent 60/992,079 pending and a patent $61 / 693861$ pending; E.K.S. reports that he received honoraria and consulting fees from Merck and grant support and consulting fees from GlaxoSmithKline; G.R.W. reports personal fees from GlaxoSmithKline, Merck, and Intellent Market Research outside the submitted work and his spouse works for Merck Research Laboratories and; H.H. reports receiving grants or has pending grants with AZE Inc., Toshiba Medical Inc., and Cannon Inc. G.M.H. reports consulting fees from Medna L.L.C., and the George Lehman Group, and advisory board work for "Patients Like Me" and Genentech. The remaining authors report no conflicts of interest with the submitted work.

\section{Authors' contributions}

All authors have read and approved the final version of the manuscript. The following authors conceived of the study: CRK, TJD, GRW, HH, GMH; participated in the acquisition of the clinical data: TA, OEZ, IEE, MK, YO, EKS, IOR, GRW, GTO, HH; the acquisition and analysis of the genetic data: WG, JD, $J C L, D A S, G T O, G M H$; statistical analysis and interpretation of the clinical data: CRK, TJD, WG, JD, JCL, EKS, GRW, GTO, HH, GMH; provided administrative, technical, or material support: OEZ, IEE, JCR, RSJE, AAD, IOR, GRW; participated in the drafting of the manuscript: CRK, TA, TJD, WG, JD, JCL, OEZ, IEE, MK, YO, JCR, RSJE, AAD, DJL, DAS, EKS, IOR, GRW, GTO, HH, GMH; and guarantor of the manuscript: $\mathrm{GMH}$.

\section{Acknowledgements}

Dr. Nishino is supported by NIH Grant Number: K23 CA157631. Dr. San José Estépar is supported by NIH Grant Numbers: K25 HL104085, R01 HL116931 and R01 HL116473. Dr. Lederer is supported by NIH Grant Numbers: R01 HL103676 and R01 HL114626. Dr. Schwartz is supported by the National Heart, Lung and Blood Institute (R01-HL095393, R01-HL097163, P01-

HL092870, and RC2-HL101715) and the Veterans Administration 
(1101BX001534). Dr. Silverman is supported by NIH Grant Numbers: R01 HL089856, P01 HL105339, and P01 HL114501. Dr. Rosas is supported by NIH Grant Number: U01 HL105371 and P01 HL114501. Dr. Washko is supported by NIH Grant Number: R01 HL116473, R01 HL107246 and P01 HL114501. Dr. Hunninghake is supported by NIH Grant Number: U01 HL105371, P01 HL114501, and R01 HL111024. This work was partially supported by the NHLBl's Framingham Heart Study contract: N01-HC-25195 (FHS) and by R01 HL089897 and R01 HL089856 (COPDGene) and R01 HL111024. This paper is subject to the $\mathrm{NIH}$ public access policy: http:// publicaccess.nih.gov.

\section{Author details}

${ }^{1}$ From the Pulmonary and Critical Care Division, Brigham and Women's Hospital, Harvard Medical School, Boston, MA, USA. ²Department of Radiology, Brigham and Women's Hospital, Boston, MA, USA. ${ }^{3}$ Department of Biostatistics, Boston University School of Public Health, Boston, MA, USA. ${ }^{4}$ The National Heart, Lung, and Blood Institute's Framingham Heart Study, Framingham, Massachusetts, Boston, MA, USA. ${ }^{5}$ Department of Medicine, Boston University, Boston, MA, USA. ${ }^{6}$ Department of Neurology, Boston University, Boston, MA, USA. ${ }^{7}$ Center for Pulmonary Functional Imaging, Brigham and Women's Hospital, Boston, MA, USA. ${ }^{8}$ Channing Division of Network Medicine, Brigham and Women's Hospital, Boston, MA, USA. ${ }^{9}$ Surgical Planning Laboratory, Department of Radiology, Brigham and Women's Hospital, Boston, MA, USA. ${ }^{10}$ Department of Pulmonary Diseases, Pontificia Universidad Católica de Chile, Santiago, Chile. " Division of Pulmonary and Critical Care, College of Physicians and Surgeons, Columbia University, New York, NY, USA. ${ }^{12}$ Department of Epidemiology, Mailman School of Public Health, Columbia University, New York, NY, USA.

${ }^{13}$ Department of Medicine, University of Colorado, Denver, CO, USA. ${ }^{14}$ Pulmonary Center, Department of Medicine, Boston University School of Medicine, Boston, MA, USA.

\section{Received: 23 February 2015 Accepted: 12 October 2015} Published online: 29 October 2015

\section{References}

1. American Thoracic Society/European Respiratory Society International Multidisciplinary Consensus Classification of the Idiopathic Interstitial Pneumonias. This joint statement of the American Thoracic Society (ATS), and the European Respiratory Society (ERS) was adopted by the ATS board of directors, June 2001 and by the ERS Executive Committee, June 2001. Am J Respir Crit Care Med. 2002;165:277-304.

2. King Jr TE, Tooze JA, Schwarz MI, Brown KR, Cherniack RM. Predicting survival in idiopathic pulmonary fibrosis: scoring system and survival model. Am J Respir Crit Care Med. 2001;164:1171-81.

3. Richeldi L, du Bois RM, Raghu G, Azuma A, Brown KK, Costabel U, et al. Efficacy and safety of nintedanib in idiopathic pulmonary fibrosis. N Engl J Med. 2014;370:2071-82.

4. King Jr TE, Bradford WZ, Castro-Bernardini S, Fagan EA, Glaspole I, Glassberg MK, et al. A phase 3 trial of pirfenidone in patients with idiopathic pulmonary fibrosis. N Engl J Med. 2014;370:2083-92.

5. Hunninghake GM. A new hope for idiopathic pulmonary fibrosis. N Engl J Med. 2014;370:2142-3.

6. Putman RK, Rosas IO, Hunninghake GM. Genetics and early detection in idiopathic pulmonary fibrosis. Am J Respir Crit Care Med. 2014;189:770-8.

7. Tsushima K, Sone S, Yoshikawa S, Yokoyama T, Suzuki T, Kubo K. The radiological patterns of interstitial change at an early phase: over a 4-year follow-up. Respir Med. 2010;104:1712-21.

8. Sverzellati N, Guerci L, Randi G, Calabro E, La Vecchia C, Marchiano A, et al. Interstitial lung diseases in a lung cancer screening trial. Eur Respir J. 2011;38:392-400.

9. Washko GR, Hunninghake GM, Fernandez IE, Nishino M, Okajima Y, Yamashiro $T$, et al. Lung volumes and emphysema in smokers with interstitial lung abnormalities. N Engl J Med. 2011;364:897-906.

10. Hunninghake GM, Hatabu H, Okajima Y, Gao W, Dupuis J, Latourelle JC, et al. MUC5B promoter polymorphism and interstitial lung abnormalities. N Engl J Med. 2013;368:2192-200.

11. Lederer DJ, Enright PL, Kawut SM, Hoffman EA, Hunninghake G, van Beek EJ, et al. Cigarette smoking is associated with subclinical parenchymal lung disease: the Multi-Ethnic Study of Atherosclerosis (MESA)-lung study. Am J Respir Crit Care Med. 2009;180:407-14.

12. Doyle TJ, Washko GR, Fernandez IE, Nishino M, Okajima Y, Yamashiro T, et al. Interstitial lung abnormalities and reduced exercise capacity. Am J Resp Crit Care Med. 2012;185(7):756-62.

13. Raghu G, Collard HR, Egan JJ, Martinez FJ, Behr J, Brown KK, et al. An official ATS/ERS/JRS/ALAT statement: idiopathic pulmonary fibrosis: evidence-based guidelines for diagnosis and management. Am J Respir Crit Care Med. 2011;183:788-824.

14. Jin GY, Lynch D, Chawla A, Garg K, Tammemagi MC, Sahin H, et al. Interstitial lung abnormalities in a CT lung cancer screening population: prevalence and progression rate. Radiology. 2013;268:563-71.

15. Fingerlin TE, Murphy E, Zhang W, Peljto AL, Brown KK, Steele MP, et al. Genome-wide association study identifies multiple susceptibility loci for pulmonary fibrosis. Nat Genet. 2013;45:613-20.

16. Ando K, Sekiya M, Tobino K, Takahashi K. Relationship between quantitative CT metrics and pulmonary function in combined pulmonary fibrosis and emphysema. Lung. 2013;191:585-91.

17. Kim HG, Tashkin DP, Clements PJ, Li G, Brown MS, Elashoff R, et al. A computer-aided diagnosis system for quantitative scoring of extent of lung fibrosis in scleroderma patients. Clin Exp Rheumatol. 2010;28:S26-35.

18. Yoon RG, Seo JB, Kim N, Lee HJ, Lee SM, Lee YK, et al. Quantitative assessment of change in regional disease patterns on serial HRCT of fibrotic interstitial pneumonia with texture-based automated quantification system. Eur Radiol. 2013;23:692-701.

19. Kim WJ, Silverman EK, Hoffman E, Criner GJ, Mosenifar Z, Sciurba FC, et al. $\mathrm{CT}$ metrics of airway disease and emphysema in severe COPD. Chest. 2009;136:396-404.

20. Washko GR, Lynch DA, Matsuoka S, Ross JC, Umeoka S, Diaz A, et al. Identification of early interstitial lung disease in smokers from the COPDGene Study. Acad Radiol. 2010;17:48-53.

21. Uh HW, Wijk HJ, Houwing-Duistermaat JJ. Testing for genetic association taking into account phenotypic information of relatives. BMC Proc. 2009;3 Suppl 7:S123.

22. R Development Core Team. R: A language and environment for statistical computing. Vienna, Austria; 2009.

23. Paul J, Krauss B, Banckwitz R, Maentele W, Bauer RW, Vogl TJ. Relationships of clinical protocols and reconstruction kernels with image quality and radiation dose in a 128-slice CT scanner: study with an anthropomorphic and water phantom. Eur J Radiol. 2012:81:e699-703.

24. Seibold MA, Wise AL, Speer MC, Steele MP, Brown KK, Loyd JE, et al. A common MUC5B promoter polymorphism and pulmonary fibrosis. N Engl J Med. 2011;364:1503-12.

25. Zhang Y, Noth I, Garcia JG, Kaminski N. A variant in the promoter of MUC5B and idiopathic pulmonary fibrosis. N Engl J Med. 2011;364:1576-7.

26. Stock CJ, Sato H, Fonseca C, Banya WA, Molyneaux PL, Adamali H, et al. Mucin 5B promoter polymorphism is associated with idiopathic pulmonary fibrosis but not with development of lung fibrosis in systemic sclerosis or sarcoidosis. Thorax. 2013;68:436-41.

27. Borie R, Crestani B, Dieude $P$, Nunes $H$, Allanore $Y$, Kannengiesser $C$, et al. The MUC5B variant is associated with idiopathic pulmonary fibrosis but not with systemic sclerosis interstitial lung disease in the European Caucasian population. PLoS One. 2013;8, e70621.

28. Noth I, Zhang Y, Ma SF, Flores C, Barber M, Huang Y, et al. Genetic variants associated with idiopathic pulmonary fibrosis susceptibility and mortality: a genome-wide association study. Lancet Respir Med. 2013;1:309-17.

29. Hartley PG, Galvin JR, Hunninghake GW, Merchant JA, Yagla SJ, Speakman $\mathrm{SB}$, et al. High-resolution CT-derived measures of lung density are valid indexes of interstitial lung disease. J Appl Physiol (1985). 1994;76:271-7.

30. Paul NS, Kashani H, Odedra D, Ursani A, Ray C, Rogalla P. The influence of chest wall tissue composition in determining image noise during cardiac CT. AJR Am J Roentgenol. 2011;197:1328-34.

31. Rosas 1O, Yao J, Avila NA, Chow CK, Gahl WA, Gochuico BR. Automated quantification of high-resolution $\mathrm{CT}$ scan findings in individuals at risk for pulmonary fibrosis. Chest. 2011;140:1590-7.

32. Zavaletta VA, Bartholmai BJ, Robb RA. High resolution multidetector CT-aided tissue analysis and quantification of lung fibrosis. Acad Radiol. 2007; 14:772-87.

33. Uppaluri R, Hoffman EA, Sonka M, Hunninghake GW, McLennan G. Interstitial lung disease: a quantitative study using the adaptive multiple feature method. Am J Respir Crit Care Med. 1999;159:519-25. 
34. Crapo RO, Morris AH, Gardner RM. Reference spirometric values using techniques and equipment that meet ATS recommendations. Am Rev Respir Dis. 1981;123:659-64.

35. Stocks J, Quanjer PH. Reference values for residual volume, functional residual capacity and total lung capacity. ATS workshop on lung volume measurements. Official statement of the European respiratory society. Eur Respir J. 1995;8:492-506.

Submit your next manuscript to BioMed Central and take full advantage of:

- Convenient online submission

- Thorough peer review

- No space constraints or color figure charges

- Immediate publication on acceptance

- Inclusion in PubMed, CAS, Scopus and Google Scholar

- Research which is freely available for redistribution 